\section{Authors' response: single versus combination antibiotic therapy in adults hospitalised with community acquired pneumonia}

We thank van der Eerden $e t a l^{1}$ for their interest in our work ${ }^{2}$. We agree that pathogen-directed therapy (PDT) is desirable in adults with community-acquired pneumonia (CAP) admitted to hospital. In their report, the authors claim that microbiological investigations including sputum and pleural fluid Gram stain can be obtained 'within $2 \mathrm{~h}$ of admission $24 \mathrm{~h}$ a day'. However, in practice, the time required to obtain results from microbiological investigations is usually significantly longer in most hospitals. Greater use of point-of-care tests has the potential to reduce the time to a microbiological diagnosis. However, the cost-effectiveness of such an approach has not yet been adequately established and the limited sensitivity of currently available microbiological tests further compounds this issue. In the meantime, most adults hospitalised with CAP are treated empirically.
Their observation of higher mortality in adults receiving empirical therapy in the intensive care unit compared with PDT is unexplained. The study was not powered to reliably estimate an effect on mortality in this subset of severely ill adults and data regarding the baseline characteristics of these adults between the two groups were not reported.

An adequately powered randomised controlled trial of combination $\beta$-lactam/ macrolide antibiotics versus single-agent $\beta$-lactam antibiotics given as empirical therapy in adults hospitalised with CAP will address many of the unmeasurable biases that cannot be adequately adjusted for in cohort studies.

\section{Rodrigo, W S Lim}

Department of Respiratory Medicine, Nottingham University Hospitals NHS Trust, Nottingham, UK

Correspondence to $\mathrm{Dr}$ C Rodrigo, Department of Respiratory Medicine, Nottingham University Hospitals NHS Trust, City Campus, Hucknall Road, Nottingham NG51PB, UK; chamira@doctors.org.uk

Competing interests None.

Provenance and peer review Not commissioned; internally peer reviewed.

To cite Rodrigo C, Lim WS. Thorax 2013;68:1068.

Accepted 18 July 2013

Published Online First 19 August 2013

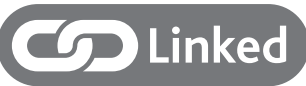

http://dx.doi.org/10.1136/thoraxjnl-2013-203994

Thorax 2013;68:1068.

doi:10.1136/thoraxjnl-2013-204221

\section{REFERENCES}

1 van der Eerden MM, Boersma WG. Single versus combination antibiotic therapy in adults hospitalised with community-acquired pneumonia. Thorax 2013;68:1069.

2 Rodrigo C, Mckeever TM, Woodhead M, et al. Single versus combination antibiotic therapy in adults hospitalised with community acquired pneumonia. Thorax 2013;68:493-5. 